\title{
Correction of bony deviation in rhinoplasty
}

\author{
Kook Hyun Kim \\ Human Plastic Surgery Clinic, Seoul, Korea
}

In rhinoplasty, osteotomy is becoming more and more frequent as a way to achieve aesthetically pleasing and functional results, as well as patient satisfaction. In procedures to correct a deviated nose, osteotomy to correct the bone plays an essential role in addition to correction of the septum and cartilage, and osteotomy can reduce the wide nose bridge and give a slightly higher appearance in Asian rhinoplasty. However, osteotomy is relatively invasive, and the nasal bones of Asians are often low and thick, so bleeding or swelling during surgery can be somewhat more severe, and a stuffy nose can occur after surgery if osteotomy is performed incorrectly. Since side effects are possible, it is necessary to have a precise understanding of the relevant anatomy and technique. Several articles have described nasal bone osteotomy in rhinoplasty, and this review article introduces the methods presented in various articles, describes indications and limitations, and reviews the relevant anatomical structures and techniques in an accurate manner. We introduce a method that can increase patients' satisfaction and the completeness of surgery through accurate osteotomy, as well as reducing the risk of side effects.

\section{Keywords Osteotomy / Rhinoplasty / Deviated nose}

\author{
Correspondence: Kook Hyun Kim \\ Human Plastic Surgery Clinic, 605 \\ Gangnam-daero, Seocho-gu, Seoul \\ 06526, Korea \\ Tel: +82-2-515-3003 \\ Fax: +82-2-515-2434 \\ E-mail: Kimkookhyun@gmail.com
}

This review article was prepared by the Korean Academic Association of Rhinoplasty Surgeons (KAARS).

Received: September 26, 2020 - Revised: October 26, $2020 \bullet$ Accepted: October 26, 2020

pISSN: 2234-6163 • elSSN: 2234-6171 • https://doi.org/10.5999/aps.2020.01921 • Arch Plast Surg 2020;47:516-521

\section{INTRODUCTION}

Although there are some cases of cartilaginous deviated nose, in which only the nasal septum and cartilage are bent, most cases of deviated nose show deviation in both the nasal bone and cartilage. Even if the deviation is only cartilaginous, the nasal septum or upper lateral cartilage cannot be aligned with the center without correctly aligning the nasal bone to the center of the joint formed by cartilage and bone. Therefore, most cases of deviated nasal septum require correction of the nasal bone- that is, osteotomy.

Osteotomy is arguably the most invasive surgical procedure performed for the nose. Therefore, osteotomy requires an accurate and precise technique due to the risk of morphological and functional complications. Conversely, safe and accurate osteotomy leads to a better prognosis and higher satisfaction.

In rhinoplasty, osteotomy is performed to close an open roof and to narrow the bony nasal pyramid or deviated nasal bone. In this paper, we present a description of how to perform corrective surgery for a deviated nose using osteotomy, and we aim to equip rhinoplasty specialists with a precise understanding of the appropriate techniques by reviewing the advantages and disadvantages of various methods and examining their indications.

\section{ANATOMY}

The nasal bone comprises the upper one-third of the nose, and contains a set of two bones that connect from the maxilla on 
both sides to the frontal bone. In the posterior aspect, they connect with the perpendicular plate of the ethmoid. Osteotomy involves detaching the nasal bone from these adjacent structures, relocating them into the desired position, and reestablishing them. In surgery, a thorough understanding of anatomy is essential. The same holds for osteotomy of the nasal bone; if surgery is performed with appropriate caution regarding the surrounding crucial anatomical structures, postoperative side effects can be minimized [1].

\section{Lacrimal sac}

The posterior part of the maxilla's frontal process meets with the lacrimal bone to form the lacrimal sac. When performing lateral osteotomy, the surgeon must be mindful of the structure. While many Asian people have small nasal bones, these bones are also relatively thick. Therefore, excessive manipulation may lead to unnecessary osteotomy, for which reason the surgeon must always be cautious while performing the operation.

\section{Keystone area}

The keystone area is where the bony septum and cartilaginous septum connect, and this refers to the copula of the distal end and the upper lateral cartilage. Their overlap typically extends for about $10 \mathrm{~mm}$, but is slightly shorter in Asians. If this part is separated completely, the stability of the middle vault may be lost.

\section{Webster's triangle}

If lateral osteotomy of the nasal bone is initiated from the bottom-most part of the pyriform aperture, this part could move inside, causing the inferior turbinate to move inside as well, which leads to nasal obstruction. Therefore, the triangle-shaped pyriform aperture, which is referred to as Webster's triangle, is preserved by initiating the osteotomy from the slightly upper part of the inferior turbinate (Fig. 1).

\section{OPERATIVE TECHNIQUES}

\section{Medial osteotomy}

Medial osteotomy is essential for the centralization of a convex and concave bony portion on both sides in a deviated nose. It may not be required if the large bony hump is adjusted, as the procedure creates an open roof. However, medial osteotomy is necessary for a deviated nose to relocate the bone into the desired position. Relocating the nasal bone with force after lateral osteotomy could cause fracture lines to appear in an undesired position. As green-stick fractures tend to return to their previous position, creating a complete fracture line through medial osteotomy is critical.

\section{Fig. 1. Webster's triangle}

Webster's triangle is highlighted in red. The triangular strut of the maxillary bone is left intact when the surgeon begins the osteotomy high on the piriform aperture.

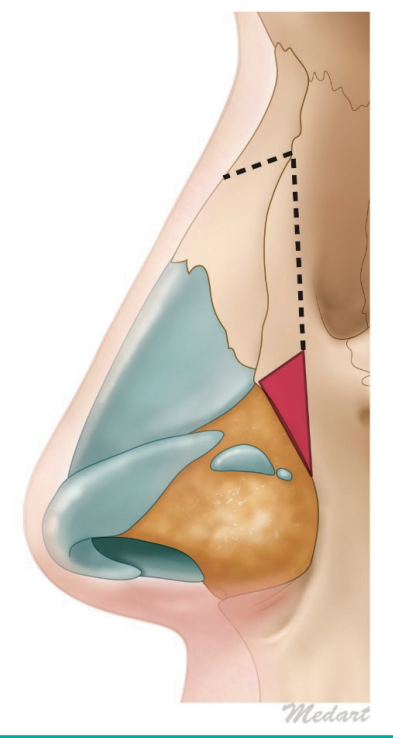

Medial osteotomy can be categorized as transverse, medial oblique, superior oblique, and paramedian based on the fracture line. An appropriate method may be implemented based on the shape of the nose, but the most cephalic extent may not reach higher than the intercanthal line (Fig. 2).

Since the thickness of the nasal bone differs in each part, it is an arduous task to create the desired fracture line following an exact line. The surgeon must be cautious of a rocker deformity if an excessively thick portion on the medial side is incised, or if the bone is cut straight without a curve [2].

\section{Lateral osteotomy}

Lateral osteotomy in the deviated nose allows relocation of the sidewall on one side of the nasal bone to the desired position after detaching it from the center, as in medial osteotomy. Lateral osteotomy can be performed inside or outside the nose with a continuous or perforating method. Whichever method is chosen, Webster's triangle needs to be preserved to prevent internal nasal valve collapse. This method of connecting the initial starting point from slightly above and then to the bottom portion, while preserving Webster's triangle, is referred to as high-to-lowto-high or high-to-low-to-low [3].

\section{Methods}

The methods of lateral osteotomy can be divided into an internal incision of the nose or an incision from the exterior of the nose, and continuous or perforating. 


\section{Fig. 2. Medial osteotomy}

Medial osteotomy categorized as transverse (A), medial oblique (B), superior oblique (C), and paramedian on the fracture line.
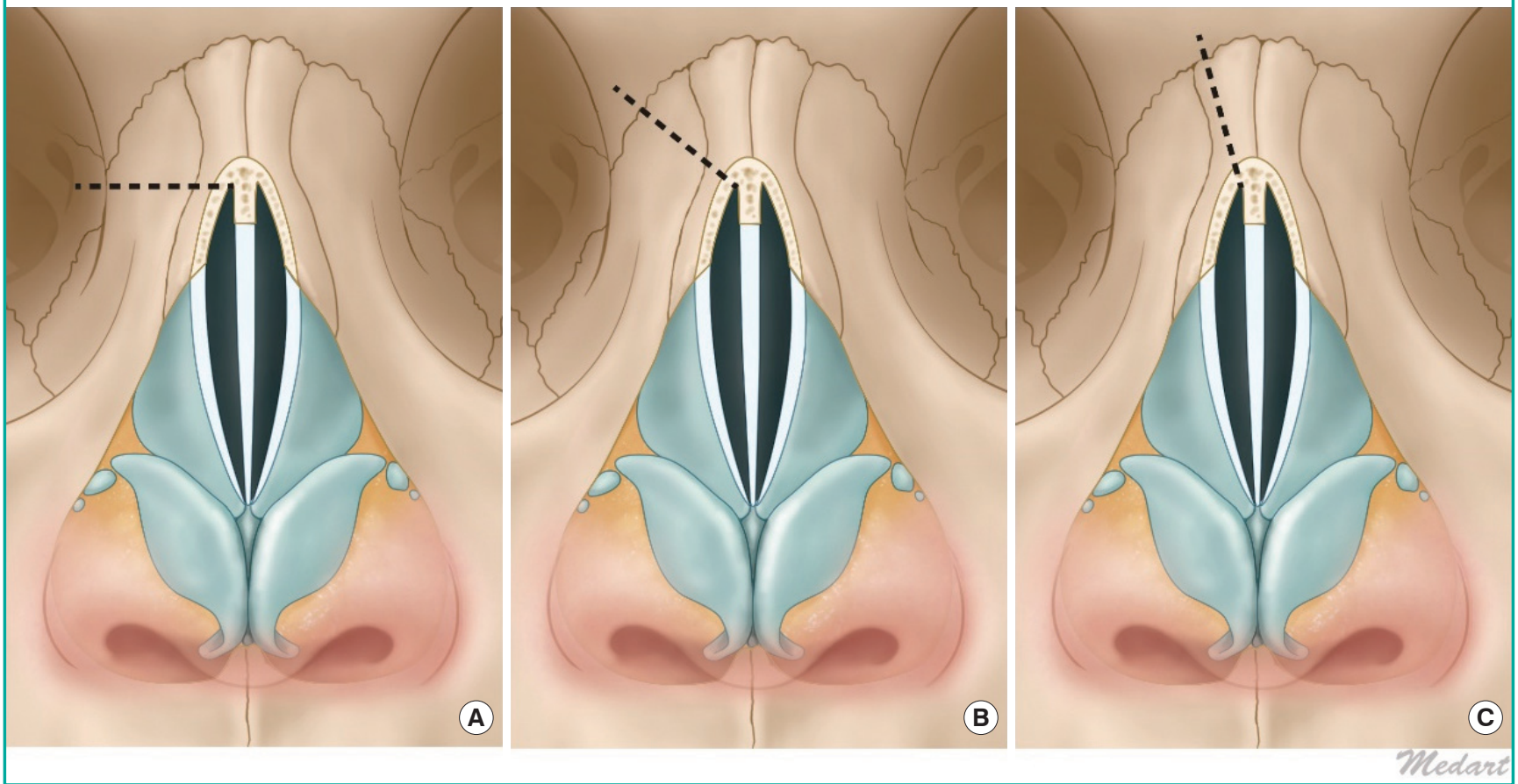

Internal continuous method

From the inside of the nose, and the bottom of the pyriform aperture, the interior turbinate is checked internally and a small incision is made. Sometimes a subperiosteal tunnel may be created, even though doing so is not absolutely necessary. After reaching the bone, as confirmed by feeling its firmness, osteotomy is performed extending 3 or $4 \mathrm{~mm}$, gradually proceeding with a two-tap technique. A slower advance is recommended, and the operator should regularly confirm that the angle of the osteotome is proceeding at the desired level using the other hand not holding the osteotome. Osteotomy is to be performed until the osteotome reaches the inner canthus. If a complete fracture does not occur after internal osteotomy, the osteotome is rotated medially to connect with the medial osteotomy line. After creating a fracture line, the surgeon confirms whether the fractured bony segment is mobile.

\section{External (percutaneous) perforating method}

The surgeon creates discontinuous perforations using a sharp osteotome, and then makes a greenstick fracture by connecting them. This method ensures the stability of the periosteum and bone by minimizing the overall damage to the periosteum. A cleanly sanitized $2 \mathrm{~mm}$ osteotome is prepared by sharpening, and then an incision is made on the medial part of the nasal bone around the inferior orbital rim, following the previously designed osteotomy line. Then, discontinuous perforating osteotomy is performed cautiously. The surgeon should advance the osteotome carefully, mindful of potential damage to the angular artery, create a greenstick fracture by applying slight pressure with both hands, insert the elevator within the nose, and carefully position the bone fragment in the desired position.

The advantage of this method is that it can improve stability after osteotomy, reduce the amount of dead space, and prevent excessive movement of the bone.

\section{Internal perforating method}

The internal perforating method involves wide dissection of the nasal envelope, and then performing osteotomy with the bone observed under direct vision. When the lower lateral cartilage, pyriform ligament, and nasal hinge area are separated, the surgeon can see the part where the base of nasal bone emerges from the pyriform aperture. The surgeon carefully separates the periosteum, exposes the bone, and then performs lateral osteotomy in the oblique direction using an osteotome, maintaining a certain distance from the medial osteotomy line and being sure to advance deliberately. While the internal perforating method requires a wider dissection of the periosteum, surgery can be done without leaving a small scar on the skin adjacent to the nose. The surgeon can perform the operation by manual palpation and visual observation, and then can create an osteotomy 


\section{Fig. 3. Lateral osteotomy}

(A-C) The level of lateral osteotomy is determined relative to the location of the proceeding osteotomy line before lateral osteotomy is performed, and it is referred to as low or high based on its relative location.
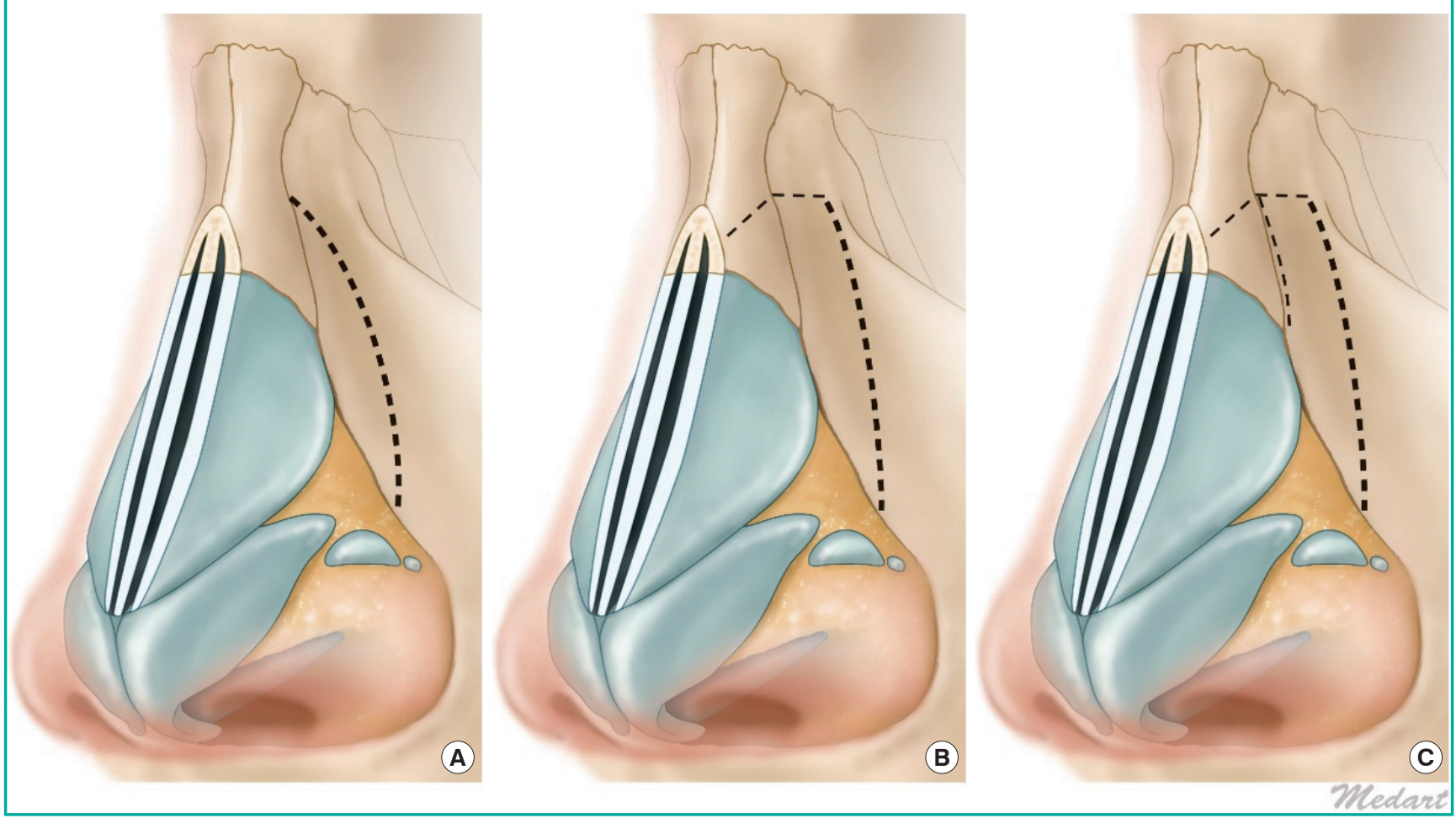

line at the precise location that the surgeon desires. Moreover, post-osteotomy stability is ensured to some extent, as osteotomy may be performed without damaging the internal side of the periosteum and the internal lining of the mucosa [4].

\section{Level}

The level is determined relative to the location of the preceding osteotomy line before lateral osteotomy is performed, and it is referred to as low or high based on its relative location. That is, the level is referred to as low if the osteotomy line runs through a lower position, or closer to the maxilla, and high if it has a higher position, closer to the dorsal line of the nasal bone. The procedure can be categorized as low-to-high, low-to-low, or double-level osteotomy, based on the sequence of levels at which it proceeds.

\section{Low-to-high}

The procedure starts from the pyriform aperture, proceeds through the intercanthal line, and then rises to a high level of the nasal dorsum. It can intersect with the medial osteotomy. If not, the bony sidewall may be mobilized through a minuscule greenstick fracture (Fig. 3A).
Low-to-low

The low-to-low technique has the same initiation point as the low-to-high technique, but the fracture line that proceeds through the intercanthal line can be finished in the lower position if the surgeon desires more mobility of the bone, or wants to move bone from other parts of the nose. If the fracture line intersects with the medial osteotomy line, the nasal bone becomes more mobile, and movement of the bone becomes more frequent as larger bones are rendered mobile (Fig. 3B).

\section{Double level}

By creating two fracture lines in the lateral sidewall, double-level lateral osteotomy is performed to remedy excessive convexity. Convexity may be controlled by performing intermediate osteotomy parallel to a low-to-low osteotomy line. The level of intermediate osteotomy could be placed through the most convex part, but ordinarily, it follows the nasal maxillary structure. Creating bony tilting through a double-level osteotomy may achieve the effect of medialization (Fig. 3C).

Regardless of the selected level, Webster's triangle must be preserved. Doing so is essential to prevent internal nasal valve collapse $[5,6]$. 


\section{Sequence of osteotomy}

In a patient with a deviated nose, the bone location may be corrected through osteotomy if one side is convex and the other side is concave. While some cases only exhibit bony deviation, most cases also show septal deviation and deviation of the upper lateral cartilage, which must be assessed carefully.

Although there is no set timing of osteotomy during surgery, if it is performed too early, swelling and bleeding may make it harder to make an accurate diagnosis during surgery. Conversely, if osteotomy is performed too late, the cartilage portion and the center may not align even after osteotomy, and the procedure may fail to achieve the aesthetic dorsal height desired by the patient. Therefore, the selection of an appropriate timing for the osteotomy procedure is crucial.

Through an open approach, first, the surgeon should expose the bone by subperiosteal dissection, and then should expose the septal cartilage through submucosal resection. The surgeon then should plan for septal deviation correction. If any hump is identified, it should be corrected first. When the septum is exposed and upper lateral cartilage is separated, the surgeon should plan the osteotomy procedure after confirming the extent of the bony deviation.

The surgeon should examine the concave side and the convex side. If one side is concave, but the other side is straight (not convex), the deviated nose may be corrected through a batten graft or camouflage graft. Some mild cases of bony deviation can be corrected through augmentation only.

If bony deviation needs to be corrected through osteotomy, the first step should be correcting the nasal bone on the concave side. To lateralize the concave nasal bone, the surgeon performs osteotomy and medial osteotomy. When the concave bone is out-fractured, a bony gap appears. Since the fractured bone may return to its original position through this gap, it should be stabilized by filling the bony gap with an extended spreader graft to prevent the fractured bone from returning to its original position.

If one side is convex, the surgeon performs medial and lateral osteotomy. If the degree of deviation is severe, the surgeon should consider bony strip resection and intermediate osteotomy.

While performing osteotomy, the complete fracture should be created in full thickness. If a greenstick fracture is formed, the bone is likely to return to its original position. The corrected bone needs to stay in the desired position after osteotomy to prevent recurrence. If a complete fracture line is not created because the medial osteotomy and lateral osteotomy do not intersect, the surgeon should create a completely fractured bony segment through percutaneous transverse osteotomy.

Even if the nasal bone on both sides moves freely, it does not maintain a straight position after surgery. The surgeon needs to examine the correction of the nasal septum through submucosal resection, the deviation of the septum at its bony portion, and hypertrophy of inferior turbinate so that the corrected bony portion does not return to its original position after surgery $[7,8]$.

\section{CONCLUSIONS}

In most cases, the treatment for deviated nose requires a twopronged approach, involving correction of both bony and cartilage portions. Therefore, it is important to harmonize the precise correction procedures in the upper lateral cartilage, septum, and turbinate.

The nasal bone of Asians is relatively small but thick. Therefore, precise osteotomy may be difficult. Moreover, many patients have an implant in their nose. Hence, using an accurate and safe procedural technique should be a high priority.

Correction of the bony portion in cases of deviated nose is an arduous process. However, in most cases, a satisfactory outcome is unachievable without the correction of bony deviation. Moreover, since osteotomy is one of the most invasive surgical procedures for the nose, having a precise understanding of the relevant anatomical structures helps to perform osteotomy precisely and to ensure that the bone is located in the desired position. Furthermore, understanding the advantages and disadvantages of various osteotomy procedures could contribute to improved prognoses by enabling the surgeon to perform procedures suitable for an individual patient.

\section{NOTES}

\section{Conflict of interest}

No potential conflict of interest relevant to this article was reported.

\section{ORCID}

Kook Hyun Kim ～https://orcid.org/0000-0001-8098-0464

\section{REFERENCES}

1. Tebbetts JB. Osteotmies. In: Tebbetts JB, editor. Primary rhinoplasty. St. Louis: Elsevier Mosby; 2008. p. 211.

2. Rohrich RJ, Adams WP Jr, Ahmad J. Nasal osteotomies. In: Rohrich RJ, Adams WP Jr, Ahmad J, et al., editors. Dallas rhinoplasty: nasal surgery by the masters. 3rd ed. Hoboken: CRC Press; 2014. p. 249-274.

3. Guyuron B. Variations in nasal osteotomy: consequences and technical nuances. In: Guyuron B, editor. Rhinoplasty. 
Edinburgh: Elsevier; 2012.p. 133.

4. Rho BI, Lee IH, Park ES. Visible perforating lateral osteotomy: internal perforating technique with wide periosteal dissection. Arch Plast Surg 2016;43:88-92.

5. Bloom JD, Immerman SB, Constantinides M. Osteotomies in the crooked nose. In: Sclafani AO, editor. Rhinoplasty the experts' reference. New York: Thieme; 2015. p. 134.

6. Toriumi DM, Becker DG. Osteotomies. In: Toriumi DM,
Becker DG, editors. Rhinoplasty dissection manual. Philadelphia: Lippincott Williams \& Wilkins; 1999. p. 67.

7. Suh MK. Deviated nose correction and functional rhinoplasty. In: Suh MK, editor. Atlas of Asian rhinoplasty. Singapore: Springer; 2018. p. 669.

8. Toriumi DM. Managing the nasal profile and bony nasal vault. In: Toriumi DM, editor. Structure rhinoplasty. Vol 1. St. Louis: Quality Medical Publishing; 2019. p. 93. 